\title{
Etude de la désorption des éléments nutritifs de certains matériaux agro- géologiques du Cameroun
}

\author{
Alexandre NONO ${ }^{1^{*}}$, Antoine MVONDO ZE ${ }^{2}$ et Samuel KEUATSOP KOUAM ${ }^{1}$ \\ ${ }^{1}$ Département des Sciences de la Terre, Faculté des Sciences, Université de Dschang, BP 67 Dschang, \\ Cameroun. \\ ${ }^{2}$ Département des Sciences du Sol, Faculté d'Agronomie et des Sciences Agricoles, Université de Dschang, \\ BP 222, Dschang, Cameroun. \\ *Auteur correspondant, E-mail : alex_nono2000@yahoo.fr ; Tel : (237) 77649231.
}

\section{RESUME}

Un essai d'équilibration et une étude de la désorption en quatre semaines par la méthode de Snoek des éléments minéraux des marnes de Kompina, des cendres volcaniques de Foumbot et des brèches volcaniques de Fongo-Tongo, ont été initiés dans l'objectif de pouvoir estimer les vitesses de libération des éléments dans le sol afin de pouvoir déterminer les périodes d'épandage des poudres de roches par rapport aux semis. Lors de l'équilibration des cendres, les éléments majeurs ont été libérés plus rapidement, comparées aux autres roches, pendant les deux premières semaines et ensuite la désorption a été progressive les deux dernières semaines. La libération des éléments nutritifs par les différentes roches est dans l'ensemble proportionnelle au temps avec des libérations maximales à la deuxième semaine, et inversement proportionnelle aux doses avec des

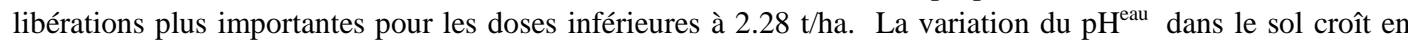
fonction du temps et des doses pour les trois types de roches alors que celle du $\mathrm{pH}^{\mathrm{kcl}}$ montre une croissance assez nette seulement pour les marnes. L'altération des trois roches étudiées a été étalée sur les quatre semaines de l'observation avec une grande aptitude des brèches à être utilisées comme fertilisant dans les systèmes diversifiés (apport d'un flux élevé en $\mathrm{P}$ et $\mathrm{Ca}$ ), les marnes pouvant être recommandées pour les cultures à cycle court puisque les éléments sont assez vite libérés à la première semaine.

(C) 2010 International Formulae Group. All rights reserved.

Mots clés : Matériaux agro-géologiques, essai d'équilibration, libération progressive, re-minéralisation, potentiel fertilisant.

\section{INTRODUCTION}

Le Cameroun comme tous les autres pays de l'Afrique subsaharienne figure parmi ceux qui enregistrent les taux d'épuisement des nutriments les plus élevés (Stoorvogel et Smaling, 1990). Selon le Centre international pour la fertilité des sols et le développement agricole (IFDC), l'Afrique perd chaque année 8 millions de tonnes métriques d'éléments nutritifs du sol et plus de 95 millions d'hectares de terre ont été dégradés au point de réduire de manière significative la productivité (Henao et Baanante, 2006). Selon des estimations, au moins $85 \%$ des pays africains souffrent d'un prélèvement des nutriments de plus de $30 \mathrm{~kg}$ par hectare et par an et $40 \%$ des pays subissent des pertes de plus de $60 \mathrm{~kg}$ de nutriments par hectare et par an (Banque Mondiale, 2006 ; Henao et Baanante, 2006). De même, il ressort de 
l'Évaluation Mondiale de la Dégradation des Sols (GLASOD) que la superficie des sols dégradés s'établit à environ 494 millions d'hectares en Afrique (Oldeman et al., 1991 ; Batjes, 2001). Toujours selon les estimations, $65 \%$ des terres agricoles de l'Afrique subsaharienne sont dégradées à cause de l'érosion hydrique du sol, et de la dégradation chimique et physique (Oldeman et al., 1991 ; PNUE, 1992 ; Scherr et Yadav, 1996).

Des pertes d'une telle ampleur imposent la prise de certaines mesures, notamment la reconstitution de la fertilité des sols, l'utilisation accrue d'engrais inorganiques et un recyclage plus efficient de la biomasse dans le système agricole. Au regard des problèmes susmentionnés, Sanchez et al. (1997) ont conclu que l'épuisement de la fertilité des sols dans les petites exploitations agricoles constitue la principale cause biophysique de la baisse de la production alimentaire par habitant en Afrique. Aussi, la reconstitution de la fertilité des sols doit-elle être considérée comme un investissement dans le capital de ressources naturelles.

$\mathrm{Si}$ l'utilisation excessive d'engrais inorganiques et organiques est à l'origine de la contamination environnementale dans certaines régions des pays développés, l'apport insuffisant de nutriments et la mauvaise gestion du sol ainsi que la rudesse des conditions climatiques ont contribué à la dégradation des sols en Afrique subsaharienne. L'intensité moyenne d'utilisation des engrais en Afrique subsaharienne, s'établit à environ $9 \mathrm{~kg} / \mathrm{ha}$ (FAO, 1998). Cette utilisation a généralement été très élevée dans les pays de l'Afrique australe $(16 \mathrm{~kg} / \mathrm{ha})$ et de l'Est $(8 \mathrm{~kg} / \mathrm{ha})$ et très faible dans les pays soudano-sahéliens (4 $\mathrm{kg} / \mathrm{ha}$ ) et en Afrique centrale ( $3 \mathrm{~kg} / \mathrm{ha}$ ) (Kelly, 2006). Les études diagnostiques relatives à l'utilisation des engrais en Afrique ont révélé que celle-ci est faible sur le continent, par comparaison aux pays développés, pour quatre raisons interdépendantes (Kherallah et al., 2002 ; Kelly, 2006) :

- les faibles rentabilités de l'utilisation des engrais en raison des conditions agro- climatiques et des méthodes culturales actuelles;

- l'absence d'informations sur les engrais parmi les distributeurs, agriculteurs et agents de vulgarisation, notamment les informations sur les prix et les meilleures pratiques ;

- le coût élevé des engrais en raison de leur production à l'étranger, des grandes quantités unitaires et des coûts élevés du transport ;

- l'environnement politique incohérent et défavorable caractérisé par l'instabilité des politiques gouvernementales et des donateurs en matière de subvention, qui est préjudiciable à l'investissement privé.

La plupart des sols africains sont issus de l'altération superficielle millénaire du granite et autres roches métamorphiques, par les agents atmosphériques, et contiennent intrinsèquement de faibles niveaux de nutriments des plantes (Bationo et al., 2006). La fertilité des sols est très variable et il en est de même de la réaction aux intrants. La fertilité des sols est très hétérogène et présente une grande variabilité d'une exploitation agricole à l'autre, autant au niveau local que sur l'ensemble de l'Afrique (Zingore et al., 2007). En outre, bon nombre de sols réagissent faiblement lorsque les engrais sont appliqués seuls. Cette faible «efficience agronomique» peut être améliorée grâce à une gestion complémentaire de la structure du sol et des matières agro-géologiques que l'on retrouve dans certaines localités au Cameroun (Foka Towa, 2001; Nono et al., 2002 et 2008).

Les phénomènes d'altération d'une roche, quelles que soient les conditions climatiques sont favorisés par une fragmentation de la roche et conduisent à la désorption progressive des forces de liaisons intergranulaires. Il est à préciser que l'altération s'effectue au niveau du minéral, au contact eau-cristal. La fracturation des minéraux augmente la surface de ce contact et de ce fait l'altération au niveau des surfaces de fractures, les associations tétraédriques montrent des oxygènes dont les valences ne sont pas saturées. Les molécules d'eau du 
polaire s'orientent, leurs pôles négatifs sont alors dirigés vers la fracture. Entre le milieu cristallin et l'eau, ont lieu des substitutions d'ions; les ions $\mathrm{H}^{+}$prennent par exemple la place des ions $\mathrm{K}^{+}$qui deviennent des ions libres dans l'eau. Ce phénomène d'hydrolyse entraîne un déchaussement des cations dans les édifices minéraux (Dercourt et Paquet, 1995).

Le potentiel de fertilisation d'une roche dépend de sa composition chimique, de sa quantité et de sa capacité à s'altérer et à libérer les principaux éléments nutritifs $(\mathrm{Ca}$, $\mathrm{Mg}, \mathrm{K}, \mathrm{P}$, et $\mathrm{Na}$ ). Sous l'action de l'environnement, la roche s'altère progressivement et libère des éléments nutritifs vers le sol. Ces éléments peuvent alors être prélevés par la culture pour son développement. Toutefois, lorsque la libération est trop rapide, ces éléments se perdent par lessivage. Mais par contre, lorsque la libération est trop lente, les cultures peuvent ne pas en profiter au moment opportun pour leur cycle biologique (Anderson et Ingran, 1993). La connaissance du comportement des roches (vitesse d'altération, libération des éléments et la durée d'altération), permettra de synchroniser le rythme de libération de ces éléments avec le rythme de demande en éléments par les cultures, et d'en optimiser l'utilisation et les rendements.

Cette étude contribuera à pouvoir évaluer les périodes d'épandages des poudres et des semis, afin de les utiliser pour l'amélioration de la fertilité chimique ainsi que la conservation des sols et de l'environnement.

\section{MATERIEL ET METHODES}

\section{Les brèches volcaniques de Fongo-Tongo Caractéristiques}

Les brèches volcaniques sont des roches à ciment de cendre et lapillis, et à fragments de roches magmatiques avec parfois des débris de roches encaissantes. La fragmentation a des causes variées: explosions, remises en mouvements de laves à croûtes déjà figées, écroulements superficiels, (Foucault et Raoul, 1997). Les brèches de
Fongo-Tongo (Figure 1) sont assez consolidées, de couleur gris-clair et à particules micrométriques, contiennent des fragments de roche de dimensions variables (Nono et al., 2008). Ces fragments de roches sont de même nature que les trachytes formés tout autour et constituant le toit de la cave dans laquelle affleurent les brèches. Ces roches sont utilisées par les populations locales pour la fertilisation de leur sol.

\section{Compositions chimiques}

Les différentes compositions chimiques des brèches (Nono et al., 2008) sont présentées dans les Tableaux 1 à 4 .

Les valeurs du $\mathrm{pH}$ : le $\mathrm{pH}^{\text {eau }}$ est de 9,04 et le $\mathrm{pH}^{\mathrm{kcl}}$ est de 8,21.

\section{Les cendres volcaniques de Foumbot Caractéristiques}

Les cendres volcaniques sont des fragments de roches effusives projetés par les volcans, de taille inférieure à $2 \mathrm{~mm}$. Ces fragments sont constitués de magma pulvérisé et/ou de roches broyées provenant en particulier des parois de la cheminée. Elles peuvent être dispersées sur des grandes étendues et leurs dépôts donnent des roches meubles ou consolidées. Souvent blanchâtres à grises quand elles sont fraîches, elles brunissent rapidement par altération et donnent des sols fertiles (Foucault et Raoult, 1997). Les cendres volcaniques de Foumbot (Figure 1) sont meubles et non consolidées de couleur gris-sombre et sont constituées essentiellement de particules fines $(<1 \mathrm{~mm})$. Ces roches sont aussi caractérisées par la présence d'abondants fragments de roche du socle de couleur gris-clair où l'on peut identifier certains minéraux tels le quartz, les feldspaths et les pyroxènes (Nono et al., 2008).

\section{Compositions chimiques}

Les différentes compositions chimiques (Nono et al., 2008) sont présentées dans les Tableaux 5 à 7.

Les valeurs du $\mathrm{pH}:$ le $\mathrm{pH}^{\text {eau }}$ est de 6,79 et le $\mathrm{pH}^{\mathrm{kcl}}$ est de 4,09. 


\section{Les marnes de Kompina \\ Caractéristiques}

Les marnes sont des roches sédimentaires constituées d'un mélange de calcaires et d'argiles. Elles sont moins compactes que les calcaires et moins plastiques que les argiles. Ces roches sont à grains fins, happent la langue et font effervescence à l'acide dilué (HC1 à 10\%). Elles sont d'aspect terreux ou se débitent en boulettes, ou encore en plaquettes à bords arrondis. Les marnes sont abondantes dans les formations d'âge secondaire à Actuel en association avec des calcaires. Ces roches sont exploitées surtout pour les amendements des sols. Elles augmentent en effet, non seulement le $\mathrm{pH}$ du sol, mais aussi des bases échangeables. Les marnes de Kompina (Figure 1) sont des roches cohérentes, dures, denses et massives, de couleur rouille résultant de l'altération. Une grande proportion de ces roches est constituée de coquilles (bivalves) (Nono et al., 2008).

\section{Compositions chimiques}

Les différentes compositions chimiques des marnes (Nono et al., 2008) sont présentées dans les Tableaux 8 à 11. le $\mathrm{pH}^{\mathrm{kcl}}$ est de 8,22.

Les valeurs du $\mathrm{pH}: \mathrm{pH}^{\text {eau }}$ est de 7,94 et

\section{Le sol}

Le sol utilisé dans ces travaux est un sol ferralitique de la forêt du Cameroun, appartenant à la classe Fo (les ferralsols Orthiques) selon la carte des sols du Cameroun de la FAO-UNESCO. Il a été prélevé aux environs de Yaoundé (Nsimbok).

La composition chimique de ce sol est présentée dans le Tableau 12. De ce tableau, il ressort que l'acidité et la déficience en $\mathrm{N}$ et $\mathrm{P}$ semblent être les contraintes majeures de ce sol. Les réserves en éléments nutritifs y sont généralement faibles, ce qui confère à ce sol une faible fertilité naturelle. Son utilisation intensive en agriculture nécessiterait une amélioration de son statut chimique.

\section{Méthode expérimentale}

Au cours de cet essai d'équilibrage solroche, de petits pots de volume $81,64 \mathrm{~cm}^{3}$ hermétiquement fermés à la base à l'aide du ruban adhésif, ont été utilisés. Après nettoyage et séchage de ces pots, $25 \mathrm{~g}$ de sol par pot y ont été introduits. Les doses de roches introduites dans chaque pot ont été calculées en se référant à la dose de 4 t/ha appliquée par Snoek, cité par Joanna (1992), lors de ses essais en pot. Pour notre sol de densité 1,15 , les doses suivantes ont été retenues: pour $25 \mathrm{~g}$ de sol, 2,$5 ; 5 ; 10,15$; 20 ; 25 ; 30 ; 35 et $40 \mathrm{mg}$ de roche ont été progressivement appliqués. Ce qui correspond respectivement à 0,$28 ; 0,57 ; 1,14 ; 1,71$; 2,$28 ; 2,85 ; 3,42 ; 4$ et 4,57 t/ha.

54 pots ont été confectionnés à raison de 2 pots par roche ( 3 roches) et par dose ( 9 doses). Ceci en trois séries afin d'effectuer les analyses après 1 semaine, 2 semaines et 4 semaines. Ce qui fait un total de 162 pots.

Le sol et les roches ont été traités au préalable selon la méthode de Pauwels et al. (1992) à la seule différence que les roches ont été tamisées à l'aide d'un tamis de 0,2 mm de maille tel que préconisé par Hamaker cité par Joanna (1992), pour une meilleure finesse de mouture.

L'estimation de la quantité d'eau nécessaire par pot pour arrosage s'est effectuée de la manière suivante : peser un pot vide ( $8 \mathrm{~g}$ ), ensuite y introduire $25 \mathrm{~g}$ de sol. Ce qui fait un total de $33 \mathrm{~g}$. Mettre de l'eau à saturation dans ces pots et laisser pendant 12 heures tout en s'assurant que la base du pot a des petites ouvertures. Enfin, refaire la pesée $\mathrm{du}$ pot + sol + eau $(46,20 \mathrm{~g})$. La différence entre ces deux valeurs est le poids d'eau nécessaire. Dans le cas présent, ce poids est de $13,20 \mathrm{~g}$, ce qui revient à 13,20 ml d'eau.

L'arrosage des pots s'est fait tous les deux jours. La pesée des pots est d'abord effectuée, ensuite le réajustement au poids initial avec de l'eau distillée est fait, ceci pour 
Tableau 1 : Concentrations des éléments majeurs des brèches extraits à l'eau régale.

\begin{tabular}{lccccc}
\hline Eléments & Ca & Mg & K & P & Na \\
\hline Concentrations $(\mathrm{mg} / \mathrm{kg})$ & 36890 & 10770 & 36700 & 4099 & 7039 \\
\hline
\end{tabular}

Tableau 2 : Concentrations des éléments majeurs des brèches extraits avec $0.5 \mathrm{~N} \mathrm{HNO}_{3}$

\begin{tabular}{lccccc}
\hline Eléments & Ca & Mg & K & P & Na \\
\hline Concentrations $(\mathrm{mg} / \mathrm{kg})$ & 1923 & 415.5 & 3620 & 105.9 & 63.45 \\
\hline
\end{tabular}

Tableau 3 : Concentrations des éléments majeurs des brèches extraits avec acetate-EDTA.

\begin{tabular}{lccccc}
\hline Eléments & Ca & Mg & K & P & Na \\
\hline Concentrations $(\mathrm{mg} / \mathrm{kg})$ & 1896 & 293.5 & 2982 & 98.5 & 1.145 \\
\hline
\end{tabular}

Tableau 4 : Concentrations des éléments majeurs des brèches extraits à l'eau distillée.

\begin{tabular}{lccccc}
\hline Eléments & $\mathbf{C a}$ & $\mathbf{M g}$ & $\mathbf{K}$ & $\mathbf{P}$ & $\mathbf{N a}$ \\
\hline Concentrations $(\mathrm{mg} / \mathrm{kg})$ & 22.61 & 11.5 & 313.7 & 42.1 & 16.7 \\
\hline
\end{tabular}

Tableau 5 : Concentrations des éléments majeurs des cendres extraits à l'eau distillée.

\begin{tabular}{lccccc}
\hline Eléments & Ca & Mg & K & P & Na \\
\hline Concentrations $(\mathrm{mg} / \mathrm{kg})$ & 28874 & 2510 & 602.9 & 818.6 & 7854 \\
\hline
\end{tabular}

Tableau 6: Concentrations des éléments majeurs des cendres extraits avec $0.5 \mathrm{~N} \mathrm{HNO}_{3}$.

\begin{tabular}{lccccc}
\hline Eléments & Ca & Mg & K & P & Na \\
\hline Concentrations $(\mathrm{mg} / \mathrm{kg})$ & 1762 & 14.16 & 51.35 & 782.85 & 8.065 \\
\hline
\end{tabular}

Tableau 7: Concentrations des éléments majeurs des cendres extraits avec acétate-EDTA.

\begin{tabular}{lccccc}
\hline Eléments & $\mathrm{Ca}$ & $\mathrm{Mg}$ & $\mathrm{K}$ & $\mathrm{P}$ & $\mathrm{Na}$ \\
\hline Concentrations $(\mathrm{mg} / \mathrm{kg})$ & 941.5 & 1.55 & 63.35 & 6.9 & 0.015 \\
\hline
\end{tabular}

Tableau 8 : Concentrations des éléments majeurs des marnes extraits avec l'eau régale.

\begin{tabular}{lccccc}
\hline Eléments & Ca & Mg & K & P & Na \\
\hline Concentrations $(\mathrm{mg} / \mathrm{kg})$ & 36770 & 2580 & 1050 & 252.3 & 8656 \\
\hline
\end{tabular}

Tableau 9 : Concentrations des éléments majeurs des marnes extraits avec $0.5 \mathrm{~N} \mathrm{HNO}_{3}$.

\begin{tabular}{llllll}
\hline Eléments & Ca & Mg & K & P & Na \\
\hline Concentrations $(\mathrm{mg} / \mathrm{kg})$ & 1967 & 184 & 524.5 & 18.5 & 6.19 \\
\hline
\end{tabular}

Tableau 10: Concentrations des éléments majeurs des marnes extraits à l'eau distillée.

\begin{tabular}{llllll}
\hline Eléments & Ca & Mg & K & P & Na \\
\hline Concentrations $(\mathrm{mg} / \mathrm{kg})$ & 1913 & 140.5 & 377.8 & 8.4 & 3.6 \\
\hline
\end{tabular}


Tableau 11: Concentrations des éléments majeurs des marnes extraits à l'acétate-EDTA.

\begin{tabular}{llllll}
\hline Eléments & Ca & Mg & K & P & Na \\
\hline Concentrations $(\mathrm{mg} / \mathrm{kg})$ & 45.04 & 9.5 & nd & 2.63 & 0.89 \\
\hline
\end{tabular}

Tableau 12: Caractéristiques chimiques du sol utilisé dans cette étude.

\begin{tabular}{lllllllll}
\hline Eléments & $\begin{array}{l}\mathbf{N} \text { total } \\
(\mathbf{m g} / \mathbf{g})\end{array}$ & $\mathbf{p H}^{\text {eau }}$ & $\mathbf{p H}^{\mathrm{kcl}}$ & $\begin{array}{l}\mathbf{P} \\
(\mathbf{m g} / \mathbf{k g})\end{array}$ & $\begin{array}{l}\mathbf{C a} \\
(\mathbf{m g} / \mathbf{k g}\end{array}$ & $\begin{array}{l}\mathbf{N a} \\
(\mathbf{m g} / \mathbf{k g})\end{array}$ & $\begin{array}{l}\mathbf{K} \\
(\mathbf{m g} / \mathbf{k g})\end{array}$ & $\begin{array}{l}\mathbf{M g} \\
(\mathbf{m g} / \mathbf{k g})\end{array}$ \\
\hline Concentrations & 1.25 & 4.18 & 3.85 & 2.97 & 23.85 & 6.54 & 10.85 & 2.92 \\
\hline
\end{tabular}

maintenir le sol à une capacité au champ constante.

Après chaque période ( 1 semaine, 2 semaines ou 4 semaines), la série des pots correspondants est séchée à l'étuve pendant 48 heures à $45{ }^{\circ} \mathrm{C}$. Ensuite, les mesures du $\mathrm{p}^{\text {Heau }}$ et $\mathrm{p}^{\text {Hkcl }}$ sont faites, l'analyse du phosphore assimilable par la méthode Bray2 et l'extraction multi-éléments $(\mathrm{K}, \mathrm{Ca}, \mathrm{Mg}, \mathrm{Na})$ selon la méthodologie de Pauwels et al. (1992), ont été effectuées.

Les résultats obtenus au cours de cet essai ont été analysés à l'aide du logiciel statistique STATGRAPHICS pour l'étude de la désorption des éléments et de l'évolution des $\mathrm{p}^{\mathrm{H}}$ après 1 semaine, 2 semaines et 4 semaines.

\section{RESULTATS}

Les concentrations des éléments libérées par les roches ont été obtenues en faisant la différence entre les concentrations totales obtenues (tableaux des concentrations des éléments extractibles à l'eau régale) et les concentrations initiales des éléments dans le sol.

Les pourcentages libérés ont été obtenus en faisant le rapport entre les concentrations libérées et les fractions extractibles (Tableaux 13 à 20) le tout par 100.

Les pourcentages d'enrichissement du sol ont été obtenus en faisant le rapport entre les concentrations libérées et les concentrations totales le tout par 100.

\section{Le Phosphore}

Les résultats de désorption du phosphore sont illustrés par la Figure 2. Ces résultats montrent que la libération par les marnes est la plus importante de toutes les roches étudiées.

La désorption du phosphore est fonction du temps au début dans les marnes, car à la dose de $0,28 \mathrm{t} /$ ha on passe de $21.84 \%$ la première semaine à $54.37 \%$ a la fin de la deuxième semaine. Et de la deuxième à la quatrième semaine, elle est presque constante. C'est le cas à la dose de 3.42 t/ha où l'on a $5.02 \%$ à la deuxième semaine et $5.04 \%$ à la quatrième semaine.

Pour les brèches, la libération est importante à la quatrième semaine. En effet à $0.28 \mathrm{t} / \mathrm{ha}$, on passe de $7.99 \%$ à la deuxième semaine à $15.72 \%$ à la quatrième semaine. Par contre, entre la première et la deuxième semaine, la libération n'est pas importante.

Pour les cendres et quelles que soient les doses, la désorption du phosphore est presque nulle (inférieur à $1 \%$ ).

\section{Le magnésium}

Les résultats de désorption du magnésium sont illustrés par la Figure 3. Ces résultats montrent que la libération par les cendres est la plus importante de toutes les roches étudiées.

L 'essai s'est fait uniquement en deux semaines. La désorption est croissante dans 
l'ensemble en fonction du temps. La libération est importante à la première semaine pour toutes les roches. On a $33.46 \%$ à la première semaine et $43.74 \%$ à la deuxième semaine ceci à la dose de 0.57 t/ha de cendres.

\section{Le calcium}

Les résultats de désorption du calcium sont illustrés par la Figure 4. Ces résultats montrent que la libération est presque égale pour toutes les roches étudiées.

La désorption du calcium est croissante en fonction du temps. La libération est importante entre la première et la deuxième semaine pour toutes les roches. On passe par exemple de $2.65 \%$ à la première semaine à $12.1 \%$ à la deuxième semaine dans les brèches à la dose de $0.28 \mathrm{t} / \mathrm{ha}$.

\section{Le potassium}

Les résultats de désorption du potassium sont illustrés par la Figure 5. Ces résultats montrent que la libération par les cendres est la plus importante de toutes les roches expérimentales.

La désorption est croissante dans l'ensemble en fonction du temps. La libération est importante à la première semaine pour toutes les roches. On passe par exemple de $14.71 \%$ à la première semaine à $24.50 \%$ à la quatrième semaine ceci à la dose de $0.57 \mathrm{t} / \mathrm{ha}$ pour les marnes. Entre la deuxième et la quatrième semaine celle-ci n'est pas importante (souvent constante). C'est le cas pour les cendres à la dose de 1.14 t/ha où l'on a $103.13 \%$ à la deuxième et $103.16 \%$ à la quatrième semaine.

\section{Le sodium}

Les résultats de désorption du sodium sont illustrés par la Figure 6. Ces résultats montrent que la libération par les marnes est la plus importante.

La désorption est croissante en fonction du temps pour l'ensemble des roches (la libération est plus importante à la quatrième semaine). Pour les marnes, les pourcentages passent de 0 à près de $70 \%$ à la première semaine et à la deuxième semaine pour la dose de $2.28 \mathrm{t} / \mathrm{ha}$. Pour les cendres et les brèches, la libération est importante entre la deuxième et la quatrième semaine. Les pourcentages évoluent de 146.22 à la première semaine à $312.44 \%$ à la quatrième semaine, à la dose de $0.28 \mathrm{t} / \mathrm{ha}$.

Tableau 13: Valeur du $\mathrm{pH}^{\text {eau }}$ en fonction des doses de roches et du temps.

\begin{tabular}{lllll}
\hline & Semaine & $\mathbf{1}$ & $\mathbf{2}$ & $\mathbf{4}$ \\
\hline Sol initial & Doses t/ha & & & \\
\hline \multirow{4}{*}{ Marnes } & & 4,18 & & 4,52 \\
& 0,28 & 4,19 & 4,28 & 4,61 \\
& 0,57 & 4,21 & 4,36 & 4,64 \\
& 1,14 & 4,22 & 4,50 & 4,80 \\
& 1,71 & 4,37 & 4,56 & 4,86 \\
& 2,28 & 4,40 & 4,72 & 5,02 \\
& 2,85 & 4,48 & 5,00 & 5,63 \\
& 3,42 & 4,57 & 4,16 & 5,63 \\
& 4,00 & 5,18 & 5,33 & 5,67 \\
\hline \multirow{5}{*}{ Cendres } & 4,57 & 5,53 & 5,60 & 4,45 \\
& 0,28 & 4,20 & 4,31 & 4,83 \\
& 0,57 & 4,23 & 4,59 & 5,18 \\
& 1,14 & 4,25 & 4,60 & 5,25 \\
& 1,71 & 4,26 & 4,64 & 5,37
\end{tabular}




\begin{tabular}{ccccc} 
& 2,85 & 4,46 & 4,75 & 5,39 \\
& 3,42 & 4,67 & 4,78 & 5,44 \\
& 4,00 & 4,68 & 4,80 & 5,47 \\
Brèches & 4,57 & 4,80 & 4,90 & 5,48 \\
\hline & 0,28 & 4,30 & 4,53 & 4,66 \\
& 0,57 & 4,39 & 4,60 & 4,71 \\
& 1,14 & 4,53 & 4,66 & 4,79 \\
& 1,71 & 4,63 & 4,68 & 4,88 \\
& 2,28 & 4,56 & 4,76 & 4,90 \\
& 2,85 & 4,84 & 4,85 & 5,00 \\
& 3,42 & 4,85 & 4,88 & 5,10 \\
& 4,00 & 4,90 & 4,92 & 5,16 \\
\hline
\end{tabular}

Tableau 14: Valeur du $\mathrm{pH}^{\mathrm{kcl}}$ en fonction des doses de roches et du temps.

\begin{tabular}{lllll}
\hline & Semaine & $\mathbf{1}$ & $\mathbf{2}$ & $\mathbf{4}$ \\
\hline Sol initial & Doses t/ha & & & \\
\hline \multirow{4}{*}{ Marnes } & & 3,85 & & \\
& 0,28 & 3,82 & 4,07 & 4,20 \\
& 0,57 & 3,88 & 4,08 & 4,19 \\
& 1,14 & 4,12 & 4,18 & 4,34 \\
& 1,71 & 4,16 & 4,20 & 4,30 \\
& 2,28 & 4,20 & 4,24 & 4,34 \\
& 2,85 & 4,28 & 4,31 & 4,50 \\
Cendres & 3,42 & 4,54 & 4,60 & 4,61 \\
& 4,00 & 4,58 & 4,63 & 4,73 \\
& 4,57 & 4,86 & 4,90 & 4,93 \\
\hline & 0,28 & 3,80 & 4,02 & 4,04 \\
& 0,57 & 3,82 & 4,04 & 4,09 \\
& 1,14 & 3,83 & 4,05 & 4,11 \\
& 1,71 & 3,86 & 4,08 & 4,12 \\
Brèches & 2,28 & 3,88 & 4,10 & 4,13 \\
& 2,85 & 3,89 & 4,12 & 4,13 \\
& 3,42 & 3,93 & 4,16 & 4,14 \\
& 4,00 & 3,95 & 4,20 & 4,28 \\
& 4,57 & 3,99 & 4,21 & 4,33 \\
\hline & 0,28 & 3,90 & 3,92 & 4,05 \\
& 0,57 & 3,93 & 3,96 & 4,06 \\
& 1,14 & 3,96 & 3,97 & 4,06 \\
& 1,71 & 3,97 & 3,99 & 4,13 \\
& 2,28 & 4,07 & 4,07 & 4,15 \\
& 2,85 & 4,16 & 4,20 & 4,24 \\
& 3,42 & 4,19 & 4,20 & 4,26 \\
& 4,00 & 4,30 & 4,33 & 4,35 \\
& 4,57 & 4,37 & 4,43 & 4,44 \\
\hline
\end{tabular}


Tableau 15 : Concentration totale du phosphore en fonction des doses de roches et du temps.

\begin{tabular}{|c|c|c|c|c|}
\hline & Semaine & 1 & 2 & 4 \\
\hline & \multicolumn{4}{|c|}{ Doses t/ha } \\
\hline Sol initial & & 2,98 & & \\
\hline \multirow{9}{*}{ Marnes } & 0,28 & 4,11 & 5,74 & 5,78 \\
\hline & 0,57 & 4,15 & 5,94 & 6,01 \\
\hline & 1,14 & 4,18 & 5,94 & 6,07 \\
\hline & 1,71 & 4,23 & 5,98 & 6,08 \\
\hline & 2,28 & 4,25 & 6,04 & 6,14 \\
\hline & 2,85 & 4,25 & 6,08 & 6,14 \\
\hline & 3,42 & 4,28 & 6,14 & 6,15 \\
\hline & 4,00 & 4,34 & 6,15 & 638 \\
\hline & 4,57 & 4,34 & 6,22 & 6,71 \\
\hline \multirow{9}{*}{ Cendres } & 0,28 & 4,05 & 4,77 & 4,85 \\
\hline & 0,57 & 4,05 & 4,80 & 5,24 \\
\hline & 1,14 & 4,63 & 5,24 & 5,72 \\
\hline & 1,71 & 4,63 & 5,35 & 6,25 \\
\hline & 2,28 & 4,92 & 5,64 & 7,71 \\
\hline & 2,85 & 4,92 & 6,52 & 7,64 \\
\hline & 3,42 & 4,94 & 5,94 & 7,64 \\
\hline & 4,00 & 4,98 & 6,52 & 8,58 \\
\hline & 4,57 & 5,21 & 7,69 & 8,58 \\
\hline \multirow{9}{*}{ Brèches } & 0,28 & 5,22 & 5,35 & 7,64 \\
\hline & 0,57 & 5,51 & 5,94 & 7,64 \\
\hline & 1,14 & 5,72 & 7,10 & 9,51 \\
\hline & 1,71 & 5,79 & 7,68 & 9,51 \\
\hline & 2,28 & 6,38 & 8,85 & 10,44 \\
\hline & 2,85 & 6,38 & 9,44 & 10,44 \\
\hline & 3,42 & 6,68 & 10,60 & 10,51 \\
\hline & 4,00 & 7,08 & 11,78 & 10,51 \\
\hline & 4,57 & 9,28 & 12,93 & 10,51 \\
\hline
\end{tabular}

Tableau 16: Concentration totale du magnésium en fonction des doses de roches et du temps.

\begin{tabular}{|c|c|c|c|c|}
\hline & Semaine & 1 & 2 & 4 \\
\hline & \multicolumn{4}{|l|}{ Doses t/ha } \\
\hline Sol initial & & 2,92 & & \\
\hline \multirow{9}{*}{ Marnes } & 0,28 & 6,15 & 6,79 & \\
\hline & 0,57 & 6,49 & 6,95 & \\
\hline & 1,14 & 6,52 & 7,00 & \\
\hline & 1,71 & 6,66 & 7,01 & \\
\hline & 2,28 & 6,76 & 7,08 & \\
\hline & 2,85 & 6,87 & 7,10 & \\
\hline & 3,42 & 6,89 & 7,12 & \\
\hline & 4,00 & 7,38 & 4,42 & \\
\hline & 4,57 & 7,68 & 7,76 & \\
\hline \multirow{3}{*}{ Cendres } & 0,28 & 4,86 & 6,26 & \\
\hline & 0,57 & 5,62 & 6,45 & \\
\hline & 1,14 & 5,71 & 6,76 & \\
\hline
\end{tabular}




\begin{tabular}{cccc} 
& 1,71 & 5,85 & 6,78 \\
2,28 & 6,16 & 6,79 \\
2,85 & 6,18 & 6,81 \\
& 3,42 & 6,26 & 6,89 \\
& 4,00 & 6,48 & 6,95 \\
& 4,57 & 6,76 & 7,09 \\
\hline \multirow{3}{*}{ Brèches } & 0,28 & 6,10 & 6,47 \\
& 0,57 & 6,26 & 6,49 \\
& 1,14 & 6,33 & 6,52 \\
& 1,71 & 6,42 & 6,54 \\
& 2,28 & 6,50 & 6,69 \\
& 3,42 & 6,82 & 6,92 \\
& 4,00 & 6,85 & 6,94 \\
\hline
\end{tabular}

Tableau 17: Concentration totale du calcium en fonction des doses de roches et du temps.

\begin{tabular}{|c|c|c|c|c|}
\hline & Semaine & 1 & 2 & 4 \\
\hline & \multicolumn{4}{|l|}{ Doses t/ha } \\
\hline \multirow{2}{*}{ Sol initial } & & 23,85 & & \\
\hline & 0,28 & 50,25 & 102,12 & 108,69 \\
\hline \multirow[t]{8}{*}{ Marnes } & 0,57 & 53,05 & 107,55 & 116,15 \\
\hline & 1,14 & 56,00 & 108,70 & 120,67 \\
\hline & 1,71 & 57,96 & 118,60 & 143,91 \\
\hline & 2,28 & 79,73 & 123,93 & 164,00 \\
\hline & 2,85 & 81,36 & 154,71 & 164,12 \\
\hline & 3,42 & 83,96 & 154,81 & 164,12 \\
\hline & 4,00 & 87,11 & 184,35 & 194,10 \\
\hline & 4,57 & 92,83 & 194,58 & 214,14 \\
\hline \multirow[t]{9}{*}{ Cendres } & 0,28 & 38,93 & 85,05 & 101,22 \\
\hline & 0,57 & 39,10 & 85,08 & 101,85 \\
\hline & 1,14 & 39,65 & 89,45 & 102,83 \\
\hline & 1,71 & 39,80 & 90,65 & 103,85 \\
\hline & 2,28 & 41,61 & 92,58 & 105,72 \\
\hline & 2,85 & 42,62 & 93,64 & 106,28 \\
\hline & 3,42 & 42,99 & 93,84 & 106,28 \\
\hline & 4,00 & 44,28 & 94,52 & 109,43 \\
\hline & 4,57 & 44,66 & 94,96 & 110,60 \\
\hline \multirow[t]{9}{*}{ Brèches } & 0,28 & 38,11 & 88,99 & 103,65 \\
\hline & 0,57 & 43,26 & 94,87 & 106,56 \\
\hline & 1,14 & 44,85 & 99,29 & 107,01 \\
\hline & 1,71 & 45,84 & 99,73 & 114,56 \\
\hline & 2,28 & 46,87 & 100,01 & 115,63 \\
\hline & 2,85 & 49,86 & 101,91 & 118,71 \\
\hline & 3,42 & 50,57 & 103,94 & 121,08 \\
\hline & 4,00 & 51,86 & 105,95 & 124,71 \\
\hline & 4,57 & 51,87 & 106,38 & 143,95 \\
\hline
\end{tabular}


Tableau 18: Concentration totale du potassium en fonction des doses de roches et du temps.

\begin{tabular}{|c|c|c|c|c|}
\hline & Semaine & 1 & 2 & 4 \\
\hline & \multicolumn{4}{|c|}{ Doses t/ha } \\
\hline \multirow[t]{2}{*}{ Sol initial } & & 10,85 & & \\
\hline & 0,28 & 39,40 & 77,18 & 81,29 \\
\hline \multirow[t]{8}{*}{ Marnes } & 0,57 & 55,40 & 74,18 & 84,10 \\
\hline & 1,14 & 54,84 & 83,02 & 85,06 \\
\hline & 1,71 & 56,73 & 83,83 & 85,88 \\
\hline & 2,28 & 57,17 & 90,31 & 89,05 \\
\hline & 2,85 & 57,72 & 90,82 & 91,39 \\
\hline & 3,42 & 57,89 & 94,17 & 91,99 \\
\hline & 4,00 & 60,53 & 94,50 & 103,88 \\
\hline & 4,57 & 62,94 & 94,75 & 107,20 \\
\hline \multirow[t]{13}{*}{ Cendres } & 0,28 & 50,66 & 69,02 & 70,60 \\
\hline & 0,57 & 50,94 & 69,65 & 71,07 \\
\hline & 1,14 & 51,15 & 71,21 & 71,23 \\
\hline & 1,71 & 51,44 & 75,38 & 71,23 \\
\hline & 2,28 & 53,23 & 75,78 & 71,39 \\
\hline & 2,85 & 53,41 & 76,44 & 72,56 \\
\hline & 3,42 & 53,67 & 72,23 & 73,72 \\
\hline & 4,00 & 54,33 & 78,55 & 75,07 \\
\hline & 4,57 & 54,99 & 79,20 & 84,33 \\
\hline & 0,28 & 43,93 & 61,74 & 56,16 \\
\hline & 0,57 & 47,11 & 61,84 & 63,14 \\
\hline & 1,14 & 50,94 & 64,95 & 64,12 \\
\hline & 1,71 & 52,48 & 67,43 & 64,79 \\
\hline \multirow[t]{5}{*}{ Brèches } & 2,28 & 53,00 & 67,43 & 65,89 \\
\hline & 2,85 & 53,12 & 67,53 & 65,16 \\
\hline & 3,42 & 53,14 & 69,73 & 70,01 \\
\hline & 4,00 & 53,20 & 74,65 & 75,81 \\
\hline & 4,57 & 54,93 & 75,36 & 75,97 \\
\hline
\end{tabular}

Tableau 19 : Concentrations totales du sodium en fonction des doses de roches et du temps.

\begin{tabular}{lllll}
\hline & Semaine & $\mathbf{1}$ & $\mathbf{2}$ & $\mathbf{4}$ \\
\hline & Doses t/ha & & & \\
\hline Sol initial & & 6,54 & & \\
\hline \multirow{4}{*}{ Marnes } & 0,28 & 9,68 & 12,28 & 13,53 \\
& 0,57 & 9,94 & 12,81 & 13,59 \\
& 1,14 & 12,53 & 12,95 & 13,89 \\
& 1,71 & 13,22 & 12,83 & 13,97 \\
& 2,28 & 13,35 & 13,53 & 14,03 \\
& 2,85 & 14,22 & 14,42 & 15,78 \\
& 3,42 & 14,27 & 14,61 & 17,55 \\
& 4,00 & 14,37 & 15,18 & 18,53 \\
& 4,57 & 14,81 & 14,70 & 19,71 \\
\hline Cendres & 0,28 & 8,53 & 9,83 & 13,57 \\
& 0,57 & 8,75 & 9,94 & 13,95
\end{tabular}




\begin{tabular}{lllll} 
& 1,14 & 8,98 & 10,02 & 14,07 \\
& 1,71 & 9,20 & 10,73 & 14,21 \\
2,28 & 9,20 & 11,02 & 14,30 \\
& 2,85 & 9,20 & 11,84 & 14,37 \\
& 3,42 & 9,94 & 12,01 & 14,50 \\
& 4,0 & 10,48 & 12,38 & 14,65 \\
Brèches & 4,57 & 10,48 & 13,07 & 14,78 \\
\hline & 0,28 & 8,75 & 9,37 & 14,52 \\
& 0,57 & 9,98 & 9,91 & 14,54 \\
& 1,14 & 8,98 & 10,02 & 14,58 \\
& 1,71 & 8,98 & 10,39 & 14,82 \\
& 2,28 & 9,20 & 10,71 & 14,90 \\
& 2,85 & 9,69 & 10,89 & 14,93 \\
& 3,42 & 9,69 & 11,36 & 14,95 \\
& 4,00 & 10,20 & 11,62 & 15,67 \\
4,57 & 11,79 & 13,53 & 15,83 \\
\hline
\end{tabular}

Tableau 20: Fractions extractibles des éléments en fonction des doses des différentes roches.

\begin{tabular}{llllll}
\hline Doses t/ha & Ca & Mg & K & $\mathbf{P}$ & Na \\
\hline Marnes & & & & & \\
0,28 & 550,76 & 51,52 & 146,86 & 5,15 & 1,73 \\
0,57 & 1121,19 & 104,88 & 298,96 & 10,49 & 3,52 \\
1,14 & 2242,38 & 209,76 & 597,93 & 20,97 & 7,05 \\
1,71 & 3363,57 & 314,64 & 896,89 & 31,46 & 10,58 \\
2,28 & 4484,76 & 419,52 & 1195,86 & 41,95 & 14,11 \\
2,85 & 5605,95 & 524,40 & 1494,82 & 52,44 & 17,64 \\
3,42 & 6727,14 & 629,28 & 1793,79 & 62,92 & 21,16 \\
4,00 & 7868,00 & 736,00 & 2098,00 & 73,60 & 24,76 \\
4,57 & 8989,19 & 840,88 & 2396,96 & 84,08 & 28,28 \\
Cendres & & & & & \\
0,28 & 493,36 & 3,96 & 14,37 & 219,05 & 2,25 \\
0,57 & 1004,34 & 8,07 & 29,26 & 445,93 & 4,59 \\
1,14 & 2008,68 & 16,14 & 58,53 & 891,87 & 9,18 \\
1,71 & 3013,62 & 24,21 & 87,80 & 1337,80 & 13,79 \\
2,28 & 4017,36 & 32,28 & 117,07 & 1783,75 & 18,38 \\
2,85 & 5021,70 & 40,35 & 146,34 & 2229,69 & 22,98 \\
3,42 & 6026,04 & 48,42 & 175,61 & 2675,63 & 27,58 \\
4,00 & 7048,00 & 56,64 & 205,40 & 3129,40 & 32,26 \\
4,57 & 8052,34 & 64,71 & 234,66 & 3575,33 & 36,85 \\
Brèches & & & & & \\
0,28 & 538,44 & 116,34 & 1013,60 & 29,65 & 17,76 \\
1,14 & 2192,22 & 473,67 & 4126,80 & 120,72 & 72,33 \\
1,71 & 3288,33 & 710,50 & 6190,20 & 181,08 & 108,49 \\
2,28 & 4384,44 & 947,34 & 8253,60 & 241,45 & 144,66 \\
2,85 & 5480,55 & 1184,17 & 10317,00 & 301,81 & 180,83 \\
3,42 & 6576,66 & 1421,01 & 12380,40 & 362,17 & 216,99 \\
4,00 & 7692,00 & 1662,00 & 14480,00 & 423,60 & 253,80 \\
4,57 & 8788,11 & 1898,83 & 16543,40 & 483,96 & 289,90 \\
\hline & & & & &
\end{tabular}




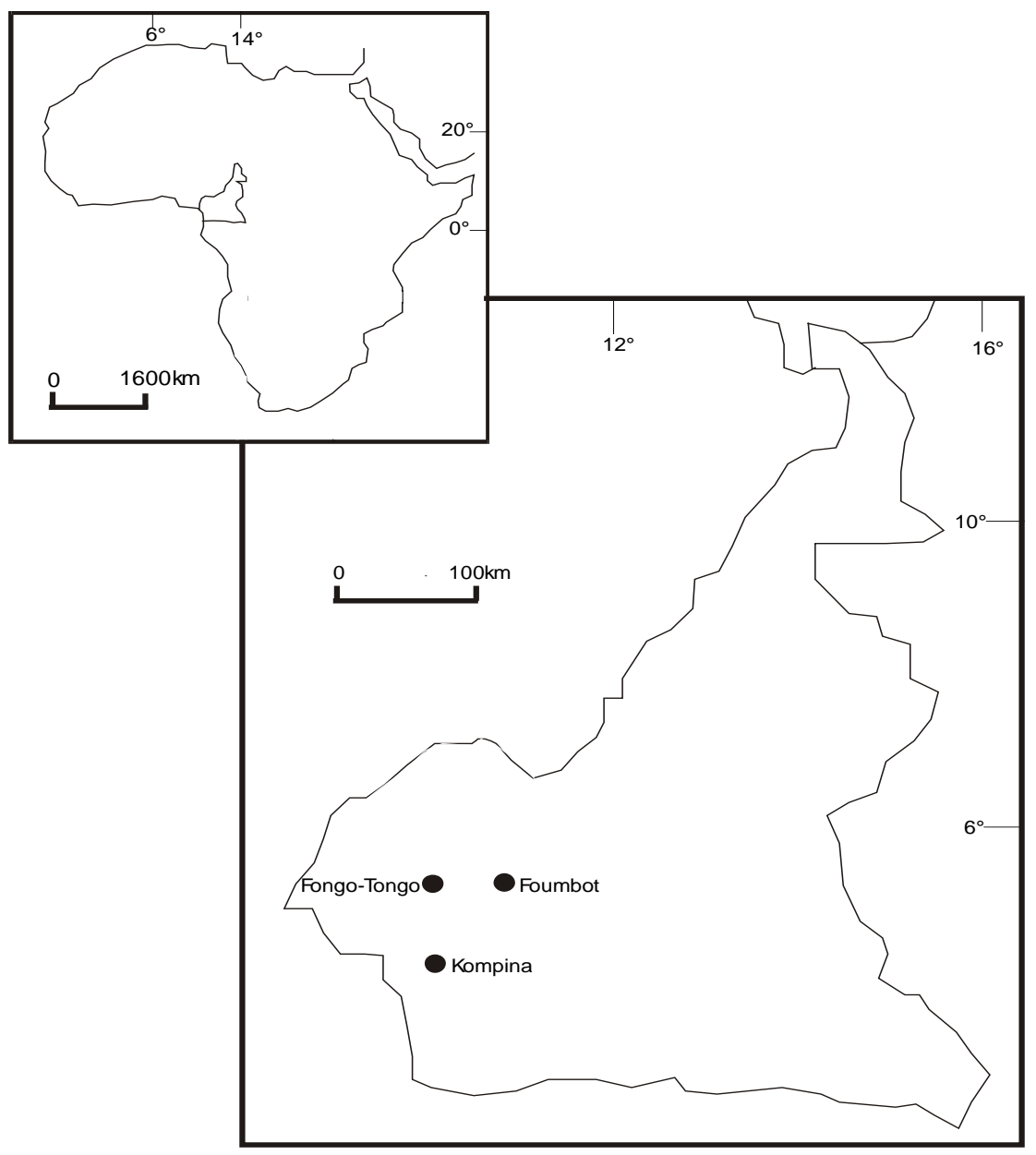

Figure 1: Localisation des secteurs de prélèvement des matériaux agro géologiques.

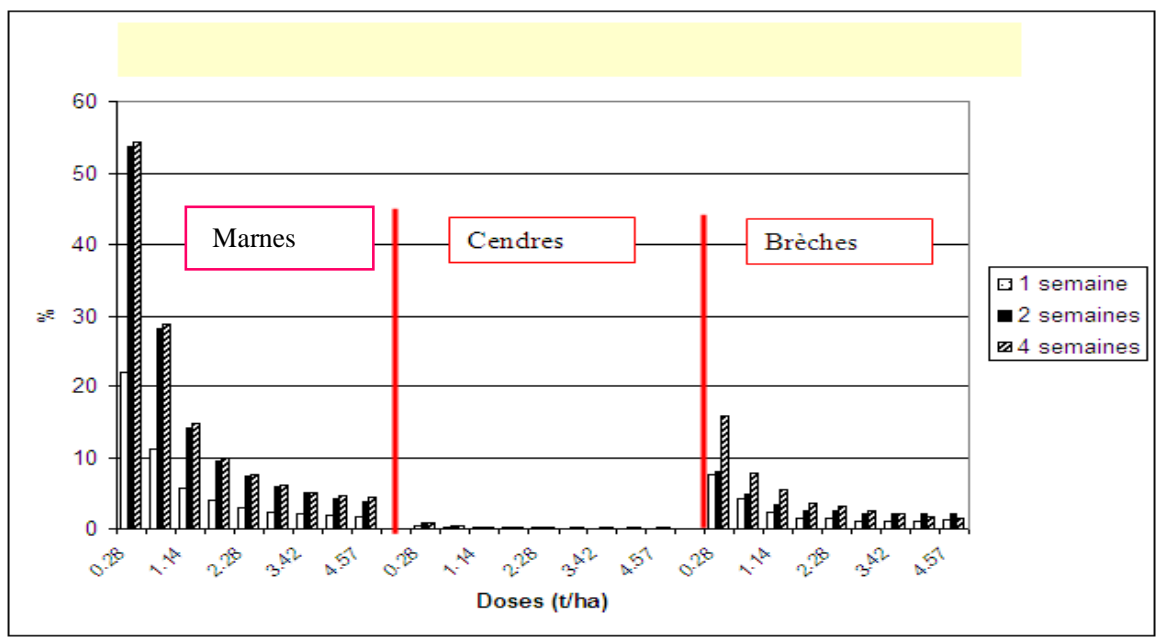

Figure 2 : Pourcentage de phosphore libéré en fonction du temps et des doses. 


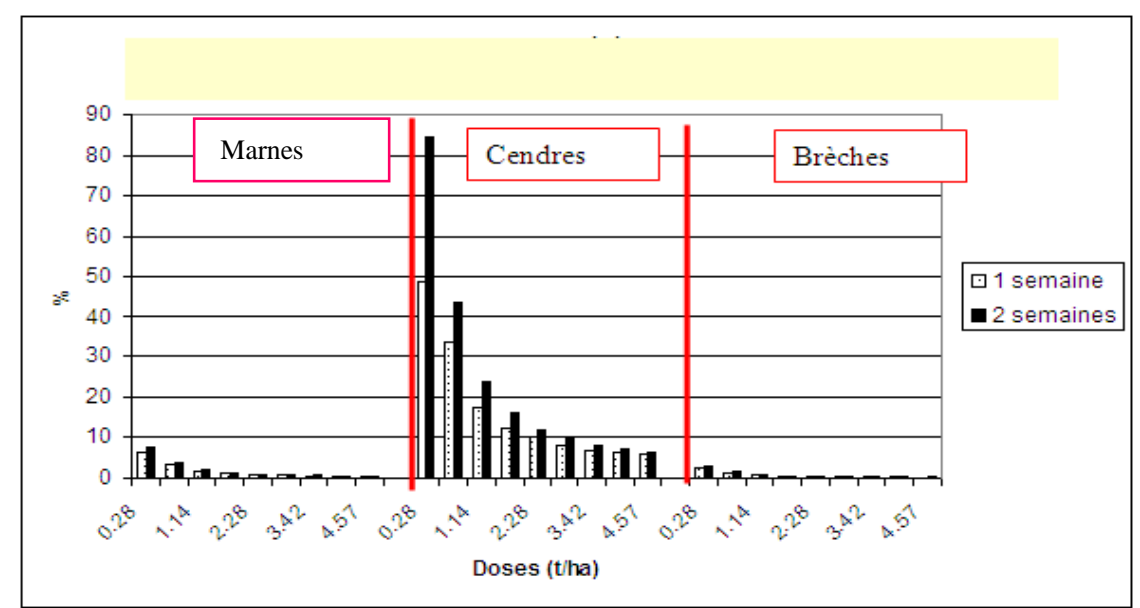

Figure 3 : Pourcentage de magnésium libéré en fonction du temps et des doses.

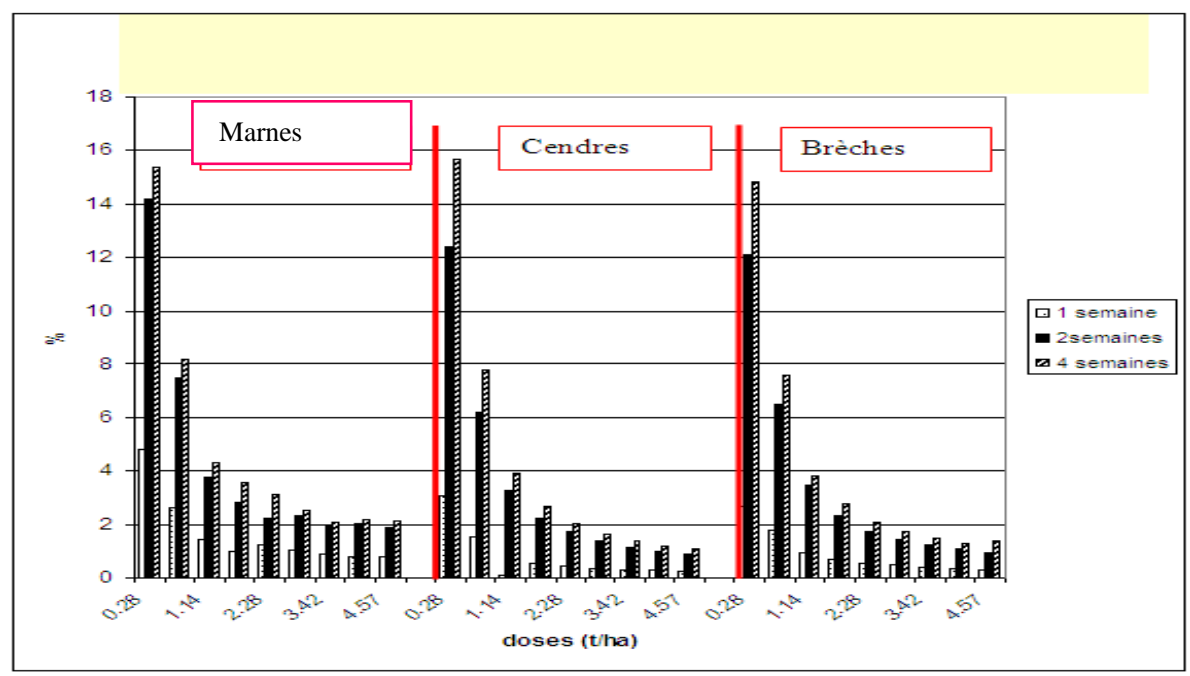

Figure 4 : Pourcentage de calcium libéré en fonction du temps et des doses.

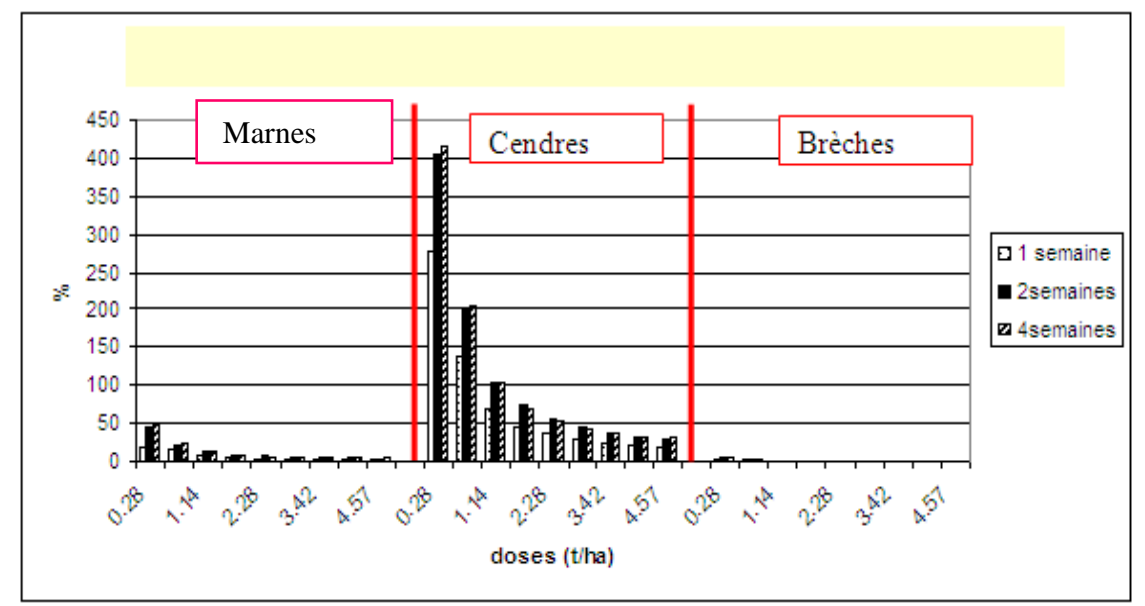

Figure 5: Pourcentage de potassium libéré en fonction des doses et du temps. 


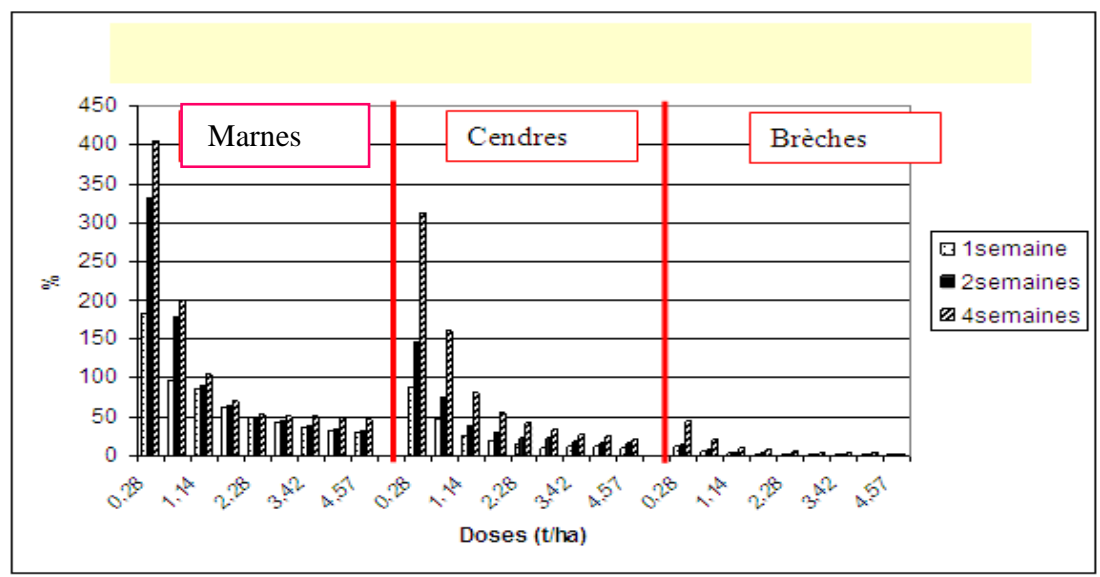

Figure 6 : Pourcentage de sodium libéré en fonction des doses et du temps.

\section{DISCUSSION}

\section{Etude de la désorption}

La désorption de tous les éléments étudiés ( $\mathrm{P}, \mathrm{Mg}, \mathrm{Ca}, \mathrm{K}, \mathrm{Na}$ ) diminue en fonction des doses croissantes pour toutes les roches étudiées. Par exemple pour $\mathrm{P}$, dans les marnes, on passe de $54.37 \%$ pour la dose de $0.28 \mathrm{t} / \mathrm{ha}$ à $4.44 \%$ pour la dose de $4.57 \mathrm{t} / \mathrm{ha}$ ceci à la quatrième semaine. En ce qui concerne le $\mathrm{Mg}$, on passe par de $7.51 \%$ pour les doses de $0.28 \mathrm{t} / \mathrm{ha}$ à $0.58 \%$ pour la dose de $4.57 \mathrm{t} / \mathrm{ha}$ ceci à la deuxième semaine pour les marnes. Dans le cas du calcium, pour les marnes à la dose de $0.28 \mathrm{t} / \mathrm{ha}$, on a $15.40 \%$ de libération et à $4.57 \mathrm{t} / \mathrm{ha}$ on a $2.12 \%$ de libération, ceci à la quatrième semaine. Le $\mathrm{K}$ montre une évolution similaire dans les cendres, car l'on passe de $51.71 \%$ à la dose de $2.28 \mathrm{t} / \mathrm{ha}$ à $31.31 \%$ à $4.57 \mathrm{t} / \mathrm{ha}$ à la quatrième semaine. Par ailleurs, il est à noter ici les libérations très importantes du potassium par les cendres pour les faibles doses. C'est le cas à $0,28 \mathrm{t} / \mathrm{ha}$ où l'on a $415.80 \%$ de libération à la quatrième semaine. Le $\mathrm{Na}$ montre que les pourcentages évoluent de 44.93 à $3.20 \%$ respectivement pour les doses de 0.28 et 4.57 $\mathrm{t} / \mathrm{ha}$ pour les brèches à la quatrième semaine. Par ailleurs, il est aussi à noter ici les libérations importantes de sodium par les marnes et par les cendres aux faibles doses (405.05\% à la dose de $0.28 \mathrm{t} / \mathrm{ha}$ à la quatrième semaine par les marnes et $325 \%$ pour les cendres). D'où la libération de $\mathrm{P}, \mathrm{Mg}, \mathrm{Ca}, \mathrm{K}$ et
$\mathrm{Na}$ par les différentes roches est proportionnelle au temps et inversement proportionnelle aux doses.

En conclusion, la libération des éléments nutritifs par les différentes roches, est proportionnelle au temps avec des libérations importantes à partir de la deuxième semaine, et inversement proportionnelle aux doses avec des libérations importantes pour les doses inférieures à 2.28 t/ha. La dissolution des éléments paraît être plus complète aux faibles doses.

\section{Evolution des pH Le $\mathrm{pH}^{\text {eau }}$}

La variation du $\mathrm{pH}^{\text {eau }}$ (tableau : valeur $\mathrm{du} \mathrm{pH}^{\text {eau }}$ ) dans le sol est croissante en fonction du temps et des doses pour les trois types de roches.

Les marnes élèvent mieux le $\mathrm{pH}^{\text {eau }}$ car après la première semaine, à la dose de 4.57 $\mathrm{t} / \mathrm{ha}$, le $\mathrm{p}^{\mathrm{H}}$ est déjà à 5.41 au moins.

Mais la constance du $\mathrm{p}^{\mathrm{H}}$, pour les cendres aux premières semaines et pour les dernières doses par rapport aux temps dans les brèches, serait due à l'effet tampon des sols. Le p $\boldsymbol{H}^{\mathrm{kcl}}$

L'évolution du $\mathrm{pH}^{\mathrm{kcl}}$ (tableau : valeur $\mathrm{du} \mathrm{pH}^{\mathrm{kcl}}$ ) est assez nette pour les marnes. On a une croissance en fonction du temps et des doses. Une élévation moins importante du $\mathrm{pH}^{\mathrm{kcl}}$ a été enregistrée pour les cendres. Et on 
note une constance du $\mathrm{pH}$ due probablement aussi à l'effet tampon du sol. Les brèches quant à elles montrent une évolution moyenne $\mathrm{du} \mathrm{pH}^{\mathrm{kcl}}$ par rapport au temps et par rapport aux doses.

\section{Potentiel fertilisant des différentes roches}

L'altération des trois roches étudiées a été étalée sur les quatre semaines de l'observation. La libération des éléments nutritifs est proportionnelle au temps et inversement proportionnelle aux doses pour toutes les roches étudiées.

Lors de l'altération des cendres, les pourcentages des éléments majeurs $(\mathrm{P}, \mathrm{K} \mathrm{Ca}$, et $\mathrm{Mg}$ ) augmentent pendant la première semaine dans le sol (plus de $25 \%$ pour tous les éléments). Ceci est une indication que les cendres pourraient être appliquées environ à une semaine avant l'optimum des besoins de la culture (sychronisation).

Les brèches ont montré une faible aptitude à libérer les éléments (Figures 2, 3, 4,5 et 6 ). Mais elles ont enrichi le sol assez fortement en phosphore $(60,99 \%$ à $0,28 \mathrm{t} / \mathrm{ha})$ et en calcium $(76,99 \%$ à 0,28 t/ha) et apporteraient un flux élevé de ces deux éléments en quatre semaines. Cette libération progressive pendant les quatre semaines de l'observation révèle une grande aptitude des brèches à être utilisées comme fertilisant dans les systèmes diversifiés.

Dans le cas des marnes, la libération des éléments a été assez rapide (Figures 2, 3, 4,5 et 6) et on note un enrichissement du sol à plus de $30 \%$ dès la première semaine pour tous les éléments nutritifs. Elles pourraient donc être recommandées pour les cultures à cycle court puisque les éléments sont assez vite libérés.

Ces résultats montrent que les trois roches peuvent contribuer différemment et de manière significative en fonction de la vitesse de libération de leurs éléments, à l'amélioration de la qualité chimique du sol, objet de la présente étude. Cette approche innovatrice permet de jeter les bases de l'étude de l'utilisation des matériaux agrogéologiques suivant le cycle des cultures.

Comparativement à d'autres roches d'intérêt agricole, les brèches de FongoTongo, les marnes de Kompina et les cendres de Foumbot ont un potentiel assez élevé. Ceci dans la mesure où ces roches après 21 jours, ont par exemple eu de meilleurs résultats que le mélange de roches (basalte + granite) proposé par Beker sur la croissance en hauteur du maïs (Joanna, 1995). En plus, les quantités d'éléments libérés par ces roches lors de l'étude cinétique (50 à 91\% pendant les 30 premières minutes d'agitation) sont comparables et souvent supérieures dans certains cas à celles obtenues par la plupart des matériaux agrogéologiques utilisés dans le monde (Nono et al., 2008).

\section{Conclusions}

Les brèches de Fongo-Tongo sont des roches dont le potentiel fertilisant n'était connu que par les populations de ce village. Cette étude a permis de montrer que comparativement aux marnes (bien connues en agriculture), le potentiel des brèches est aussi élevé. Les performances pour l'amélioration des propriétés chimiques du sol, proviennent de sa composition chimique. Cette roche est riche en phosphore, potassium et calcium. Cette étude montre également que les brèches sont une bonne candidate pour l'amélioration des sols pauvres (cas des sols acides de la zone forestière humide du Cameroun). Seulement, il serait encore nécessaire de mieux cerner le mode de gestion dans les systèmes de production, à cause de sa richesse en sodium qui peut produire des accidents de toxicité.

Les cendres ont montré un grand potentiel lors de cette étude, ce qui confirme leur utilisation depuis toujours pour l'amélioration de la fertilité des sols (Bailey, 1967).

Le potentiel des roches étudiées est évident, il serait nécessaire d'entreprendre des 
études supplémentaires pour déterminer les effets sur la croissance des plantes.

\section{BIBLIOGRAPHIE}

Anderson JM, Ingram JSI. 1993. Tropical Soil by Biology and Fertility: a Handbook of Methods. CAB International: Walling Ford, UK.

Bailey E. 1967. James Hutton: the Founder of Modern Geology. Elsevier: Amsterdam.

Decourt J, Paquet J. 1995. Géologie : Objets et Méthodes ( $9^{\mathrm{e}}$ edn). Dunod : Paris.

Banque mondiale - Région Afrique . 2006. Promoting Increased Fertilizer Use in Africa: lessons Learned and Good Practice Guidelines. Africa Fertilizer Strategy Assessment ESW Technical Report.

Batjes NH. 2001. Options for increasing carbon sequestration in West African soils: an explanatory study with special focus on Senegal. Land Degradation and Development, 12: 131-142.

Bationo A, Hartemink A, Lungu O, Naimi M, Okoth P, Smaling E, Thiombiano L. 2006. African Soils: their productivity and profitability of fertilizer use. Document de base présenté à l'occasion du Sommet africain sur les engrais, 9-13 juin 2006, Abuja, Nigeria.

FAO (Organisation des Nations unies pour l'alimentation et l'agriculture). 1998. FAOSTAT, lien relatif aux données agricoles et à l'ultilisation des terres. http:\\faostat.fao.org/default.htm.

Foka Towa R. 2001. Chemical characterization of volcanic breccias from Fong-Tongo as a potential soil amender; comparison with volcanic ash from Foumbot and marl from Kompina. Mem. Maîtrise, Univ. Dschang, p. 73.

Foucault A, Raoult JF. 1997. Dictionnaire de Géologie ( $4^{\mathrm{e}}$ édn). Masson : Paris.

Henao J, Baanante CA. 2006. Agricultural production and soil nutrient mining in Africa. Summary of IFDC technical bulletin, IFDC, Muscle Shoals, Alabama, USA.

Joanna C. 1992. Remineralise the Earth. Joanna Campe: Northampton, MA 01060, USA.

Joanna C. 1995. Remineralise the Earth (Issus n²), Joanna Campe: Northampton, MA 01060, USA.

Kelly V. 2006. Factors affecting demand for fertilizers in sub Saharan Africa. Agriculture and Rural Development, Discussion paper 23. Washington, USA, International Food Policy Research Institute.

Kherallah M, Delagde C, Gabre-Madhin E, Minot N, Johnson M. 2002. Reforming Agricultural Markets in Africa. IFPRI and Johns Hopkins University Press: Baltimore, USA.

Nono A, Omoko M, Mvondo Ze, A, Nganfi F. 2002. Possibilité d'utilisation de certains matériaux géologiques d'origine locale dans l'amendement des sols Camerounais. $19^{\text {th }}$ colloquiun of african geology. $19^{\text {th }}$ to 22th march 2002. Elljadida, Moroco.

Nono A, Mvondo Ze A, Foka Towa R. 2008. Some chemical characteristics of selected geological materials in Cameroon (Central Africa) and their potential use for crop production. Int. J. Biol. Chem. Sci., 2(1): 21-32.

Oldeman LR, Hakkeling RTA, Sombroek WG. 1991. World map of the status of human-induced soil degradation: An explanatory note. Wageningen, Pays-Bas, International Soil Reference and Information Centre, Nairobi, Kenya, United Nations Environment Programme.

Pauwels J, Mvondo Ze, A, Vanranst E, Verloo M, 1992. Manuel de laboratoire de pédagogie. Méthodes d'analyses des sols et des plantes. Equipements, gestion des Stocks de verrerie et des produits chimiques. Pub. Agricole. Univ. Dschang: 28 - 265. 
PNUE (Programme des Nations unies pour l'environnement), 1992. Atlas mondial de la désertification. Londres, Royaume-Uni, Arnold.

Sanchez PA, Shepherd KD, Soule MJ, Place FM, Mokwunye AU, Buresh RJ, Kwesiga FR, Izac AN, Ndiritu CG, Woomer PL. 1997. Soil fertility replenishment in Africa: An investment in natural resource capital. In Replenishing Soil Fertility in Africa, Buresh RJ, Sanchez PA (eds). Madison, USA, Soil Science Society of America and American Society of Agronomy, SSSA Special Publication: 130-145.

Scherr SJ, Yadav S. 1996. Land degradation in the developing world: Implications for food, agriculture, and the environment to 2020. 2020 Vision for Food, Agriculture, and Environment. Discussion Paper 14. Washington, USA, International Food Policy Research Institute.

Stoorvogel JJ, Smaling EMA. 1990. Assessment of soil nutrient depletion in Sub-Saharan Africa, 1983-2000. Report 28. Wageningen, Pays-Bas, The Winand Staring Centre for Integrated Land, Soil and Water Research (SC- DLO).

Zingore S, Murwira HK, Delve RJ, Giller KE. 2007. Soil type, management history and current resource allocation: Three dimensions regulating variability in crop productivity on African smallholder farms. Field Crops Research, 101(3): 296-305. 